\title{
Tubular Flow Reactors With First-Order Kinetics
}

\author{
R. L. Brown \\ Institute for Materials Research, National Bureau of Standards, Washington, D.C. 20234
}

(September 19, 1977)

\begin{abstract}
A method is presented for automatically calculating true first order rate constants for gas phase and wall reactions from experimentally observed decay parameters in tubular flow reactors. It includes the effects of axial and radial diffusion and Poiseuille flow.
\end{abstract}

Key words: First-order kinetics; flow reactor; gas phase kinetics; laminar flow reactor; reactor; wall reactions.

\section{Introduction}

Steady-state tubular flow reactors are widely used in chemical kinetics studies as a means of converting the reaction time into a distance measurement. One way of using this reactor is to inject one reactant into a flowing carrier gas which contains a second reactant whose concentration is much greater than that of the first. The concentration of the first reactant is then measured as a function of distance down the tube. If distance is assumed to be equal to the product of the reaction time and the average linear flow velocity of the carrier gas, then the concentration of the first species will be simply

$$
C=C_{0} e^{-k z /\langle u\rangle}
$$

where $k$ is the first-order rate constant, $\langle u\rangle$ is the average carrier flow velocity, and $C_{0}$ is the value of $C$ when the distance $z$ is zero. The position used for the $z$ origin is arbitrary since (1) shows that $k$ can be determined from the relative concentration of $C$. However, it must be fixed at some distance downstream from the injection point so that the measurements start only after the reactants are well mixed.

Unfortunately (1) is only approximate. Laminar flow exists in most experiments so that the carrier velocity will have a parabolic profile. Reactants near the tube center will travel faster than those near the wall. This will create a radial gradient in the concentration of $C$. The extent of this gradient is a complex function of the flow velocity, reaction rate, and molecular diffusion effects. An additional complication will arise if $C$ can also be destroyed by a reaction on the tube wall. This is a common occurence if $C$ is an atomic species. In spite of these complications, $C$ still exhibits an exponential decay along the tube, as long as the observations are made sufficiently far downstream from the mixing region. Instead of (1), we now have

$C(r)=C_{0}(r) e^{-k^{*} z /\langle u\rangle}$

(downstream from the mixing region)

Here, the observed decay parameter $k^{*}$ is a function of $\langle u\rangle$, the diffusion coefficient $D_{c}$ of species $C$ in the carrier gas, the true first-order rate constant $k$, and a rate constant $k_{w}$ for a first-order wall reaction; $r$ is the distance from the tube center. This equation will hold at all points sufficiently far downstream from the mixing region. How far downstream is sufficient will be discussed later. The concentration in this region exhibits a radial distribution which remains constant along the tube. To determine $k^{*}$ correctly, it is of course necessary to measure $C$ over the same range of $r$ values at each particular position along the tube.

Once $k^{*}$ has been determined it must be related to $k$, the actual first-order rate constant. This problem has been solved by Walker [1]. ${ }^{1}$ Unfortunately, the value of $k^{*}$ is given by the first positive root of a polynomial having a fairly large number of non-negligible terms. Thus, it is not possible to give a closed expression for $k$ or $k^{*}$ in terms of the other parameters. Walker has tabulated a few arrays of $k^{*}$ values corresponding to a number of experimental conditions. To use his results, however, to correct experimental $k^{*}$ values is tedious since interpolation is required. Furthermore, the accuracy of the interpolation has not been verified. The purpose of the present work is to make his method easier to use. To do this a simple computer program has been written in the form of a FORTRAN subroutine called ROOT which will calculate $k$ or $k^{*}$ when given the values of the other parameters. A number of plots are presented of $k^{*}$ versus $k$ from which other values can be obtained by a linear interpolation whose accuracy has been verified. The program can also be used to determine higher order decay terms. From these it is possible to estimate the extent of the mixing region.

Actually, Walker's solution need be used only if a wall reaction is present. When $k_{w}$ can be neglected, there exists [2-4] a very simple, and excellent approximate formula for $k^{*}$. It is

$$
\lambda^{*}=1 / 2(\sqrt{(1+4 \lambda)}-1)
$$

where $\lambda^{*}=G k^{*} /\langle u\rangle^{2}, \lambda=G k /\langle u\rangle^{2}$, and $G=D_{c}+a^{2}\langle u\rangle^{2} /$ $48 D_{c}$; a is the radius of the reactor tube. Later, a brief discussion regarding the origin of this formula will be given.

A simplified derivation of Walker's solution is presented next. This will provide the basis for the discussion of the computer program. Following that an example demonstrating its use will be presented.

${ }^{1}$ Figures in brackets indicate the literature references at the end of this paper. 


\section{Solution of Diffusion Equation With First-Order Kinetics}

The partial differential equation which describes the tubular reactor with first-order kinetics under laminar flow conditions in the steady-state is

$$
\begin{aligned}
2\langle u\rangle\left(1-r^{2} / a^{2}\right) \frac{\partial C}{\partial z} & \\
& =D_{c}\left(\frac{\partial^{2} C}{\partial r^{2}}+\frac{1 \partial C}{r \partial r}+\frac{\partial^{2} C}{\partial z^{2}}\right)-k C .
\end{aligned}
$$

The first-order rate constant $k_{w}$ for the wall reaction appears in the boundary condition,

$$
D_{c}\left(\frac{\partial C}{\partial r}\right)_{r=a}=-k_{w} C_{r=a} .
$$

From elementary kinetic theory, $k_{w}=1 / 4 \gamma \bar{c}$, where $\gamma$ is the fraction of molecules of $C$ which are destroyed on striking the surface, and $\bar{c}$ is the mean molecular speed of species $C$.

(A more accurate formula for $k_{w}$ is given in ref. 5.)

With the following dimensionless parameters, $R=r / a, Z$ $=z / a, D=D_{c} / 2 a\langle\mathrm{u}\rangle, K=a k /\langle\mathrm{u}\rangle, K_{w}=k_{w} /\langle u\rangle$, (4) and (5) become

$$
\begin{gathered}
\left(1-R^{2}\right) \frac{\partial C}{\partial Z}=D\left(\frac{\partial^{2} C}{\partial R^{2}}+\frac{1 \partial C}{R \partial R}+\frac{\partial^{2} C}{\partial Z^{2}}\right)-1 / 2 K C \\
D\left(\frac{\partial C}{\partial R}\right)_{R=1}=-1 / 2 K_{w} C_{R=1} .
\end{gathered}
$$

In terms of these parameters, Walker's dimensionless parameters are $r^{*}=R, z^{*}=Z, u=1 / 2 D, B^{2}=K / 2 D, \delta$ $=2 D / K_{w}$

The solution of (6) for the region downstream from the mixing point is

$$
C=\sum_{i=1}^{\infty} A_{i} g_{i}(R) e^{-K_{i}^{* Z}}
$$

Values of the decay parameters $K_{i}^{*}$ are fixed by the boundary condition (7). The appropriate mixture of the radial functions $g_{i}(R)$ could be determined if the radial concentration profile at the mixing point were known. However, if the measurement of $C$ is begun at a $z$ value such that $\exp \left(-K_{i}^{*}\right) \gg \exp$ $\left(-K_{i}^{*}\right)$, for $i \geqq 2$, then only the first term in (8) will be significant. The true first-order rate parameter $K$ can thus be determined from the observed decay parameter $K_{i}^{*}$ provided the functional relationship between the two can be determined.

To establish this relationship, begin by equating (2) to the first term in (8). This gives $C(r)=C_{0}(r) \exp \left(-k^{*} z /\langle u\rangle\right)=$ $\mathrm{A}_{1} \mathrm{~g}_{1}(\mathrm{R}) \exp \left(-K_{i}^{*} Z\right)=\mathrm{A} \mathrm{g}(\mathrm{R}) \exp \left(-\mathrm{K}^{*} \mathrm{Z}\right)$, which defines the dimensionless decay parameter $K^{*}=k^{*} a /\langle u\rangle$. Substituting this into (6) and (7) yields a differential equation and boundary condition which must be satisfied by $g(R)$. These are,

$$
\frac{d^{2} g}{d R^{2}}+\frac{1 d g}{R d R}+\left[K^{* 2}+\left(1-R^{2}\right) K^{*} / D-K / 2 D\right] g=0
$$

$$
\left(\frac{d g}{d R}\right)_{R=1}=-\frac{K_{w g} g_{R=1}}{2 D}
$$

This function can be expressed as a power series in even powers of $R$.

$$
g(R)=\sum_{n=0}^{\infty} B_{n} R^{2 n}
$$

The coefficients $B_{n}$ are given by

$$
\begin{aligned}
& B_{1}=-1 / 4 \alpha B_{0} \\
& B_{n}=\frac{1}{(2 n)^{2}}\left[\left(K^{*} / D\right) B_{n-2}-\alpha B_{n-1}\right]
\end{aligned}
$$

where

$$
\alpha=K^{* 2}+K^{*} / D-K / 2 D
$$

When $R=0$, we have $g=B_{0}$, so that $B_{0}$ is proportional to the concentration at the tube axis. Since only the relative concentration is of interest here, $B_{0}$ will be arbitrarily given the value unity. To derive (12) simply insert (11) into (9) and write out a few terms having different values of $n$ starting with $n=0$, and set the coefficients of like powers of $R$ equal to zero. (If the more general power series containing both even and odd powers of $R$ is used instead of (11), this procedure will show that only even powers of $R$ have non-zero coefficients.)

Equation (11) must also satisfy the boundary condition (10). Substituting it into (10) gives

$F\left(K^{*}, K, K_{w}, D\right)=\sum_{n=0}^{\infty} B_{n}\left(2 n+K_{w} / 2 D\right)=F(x)=0$.

$F$ is a function of $K^{*}, K, K_{w}$, and $D$. If the latter three quantities are specified, then the $K^{*}$ value of interest will be the first positive root of $F$ considered to be a function of $K^{*}$. Alternatively, if $K^{*}, K_{w}$, and $D$ are given, then $K$ will be a positive root of $F$, but not necessarily the smallest one. In experiments where $C$ is destroyed on the wall, $K_{w}$ can be determined by measuring $K^{*}$ in the absence of the second reactant. Then $K=0$ and (13) can be solved directly for $K_{w}$ in terms of $K^{*}$ and $D$ to give

$$
K_{w}=\frac{-2 D \sum_{n=0}^{\infty} B_{n}(2 n)}{\sum_{n=0}^{\infty} B_{n}} .
$$

The subroutine ROOT uses the Newton-Raphson method [6] to determine the roots of (13). If $x_{j}$ is an approximate 
value of $x$, then a better value $x_{j+1}$ is given by the recurrence relation

$$
x_{j+1}=x_{j}-F\left(x_{j}\right) / F^{\prime}\left(x_{j}\right)
$$

where $F^{\prime}\left(\mathrm{x}_{j}\right)$ is the derivative of $F$ with respect to $x$ at the value $x=x_{j}$. The sequence $x_{0}, x_{1}, \ldots$ will converge to the correct root if the initial value is reasonably close to the correct value.

The subroutine ROOT, shown in the appendix, requires the following calling statement,

\section{CALL ROOT(ZS,Z,ZW,D,IOPT,F,B, NB,IFLAG)}

where

$$
\begin{aligned}
& \mathrm{ZS}=K^{*} \text {, the observed first order decay parameter, } \\
& \mathrm{Z}=K \text {, the true first-order gas phase rate constant, } \\
& \mathrm{ZW}=K_{w} \text {, the first-order wall decay constant, } \\
& \mathrm{D}=D \text {, the diffusion coefficient of species } C \text { in the }
\end{aligned}
$$

If IOPT $=1$, ROOT evaluates the function $F\left(K^{*}, K, K_{w}, D\right)$ If $\mathrm{IOPT}=2$, then $K^{*}$ is calculated starting from an approximate value.

If $\mathrm{IOPT}=3, K$ is calculated from an approximate value.

If $\mathrm{IOPT}=4, K_{w}$ is calculated.

$\mathrm{F}=F\left(K^{*}, K, K_{w}, D\right)$, defined by $(13)$,

$\mathrm{B}=B_{n}$ the coefficients defined by (12). It is an array which must be dimensioned to 30 in the calling program.

$\mathrm{NB}=$ the number of terms in (13) or (14) used by ROOT for a particular calculation.

IFLAG $=1$ if the number of iterations in the NewtonRaphson procedure exceeds the number IMAX. IFLAG $=0$ otherwise.

To implement the Newton-Raphson method, ROOT calculates the derivative of $F$ from the formula

$$
F^{\prime}=\sum_{n=0}^{\infty} B_{n}^{\prime}\left(2 n+K_{w} / 2 D\right) .
$$

For IOPT $=2$, we want the derivative with respect to $K^{*}$; here, the $B_{n}^{\prime}$ are given by

$$
\begin{aligned}
B_{0}^{\prime}= & 0 \\
B_{1}^{\prime}= & -1 / 4 \alpha^{\prime} \\
B_{n}^{\prime}= & \frac{1}{(2 n)^{2}}\left(B_{n-2} / D+K^{*} B_{n-2}^{\prime} / D-\alpha^{\prime} B_{n-1}\right. \\
& \left.-\alpha B_{n-1}^{\prime}\right) \\
\alpha^{\prime}= & 2 K^{*}+1 / D .
\end{aligned}
$$

For IOPT $=3$, the derivative of $F$ with respect to $K$ is needed; in this case, the $B^{\prime}{ }_{n}$ are

$$
\begin{aligned}
& B_{0}^{\prime}=0 \\
& B_{1}^{\prime}=-1 / 4 \alpha^{\prime} \\
& B_{n}^{\prime}=\frac{1}{(2 n)^{2}}\left(K^{*} B_{n-2}^{\prime} / D-\alpha^{\prime} B_{n-1}-\alpha B_{n-1}^{\prime}\right) \\
& \alpha^{\prime}=-1 / 2 D .
\end{aligned}
$$

ROOT continues to add terms to $F$ until its absolute value changes by less than the number PREC1. Up to 30 terms are allowed by the dimension of the $B$ array, but fewer than 10 usually suffice for calculating the low order roots. (If the dimensions of $B$ are changed in ROOT, it is also necessary to change the dimensions of the array $\mathrm{BN}$ which contains the derivatives of the $B_{n}$ values.) ROOT continues iterating until the absolute value of $K^{*}$ (or $K$ ) changes by less then the number PREC2. A value of $1 \times 10^{-4}$ for both PRECl and PREC2 has been used. The maximum number of terms used for calculating $F$ is specified by the number NMAX, which must not exceed the dimensions of $B$. The values of PREC1, PREC2, NMAX, and IMAX are assigned in the DATA statement.

For ROOT to be automatic, i.e., for it to calculate $K$ or $K^{*}$, given the other parameters, it is necessary to specify suitable starting values for the Newton-Raphson calculation in terms of these parameters. When $K^{*}$ is desired, (IOPT $=$ 2 ), a satisfactory initial value is $0.9 K$. For a determination of $K(\mathrm{IOPT}=3)$, the initial value $1.2 K^{*}$ is satisfactory. These starting values have been checked and found to give convergence to the correct root over the following ranges of parameter values;

$$
\begin{aligned}
& 0 \leqq K^{*} \leqq 0.68 \\
& 0 \leqq K \leqq 0.58 \\
& 0 \leqq K_{w} \leqq 0.2 \\
& 0.01 \leqq D \leqq 1.0
\end{aligned}
$$

They may also be satisfactory outside these ranges, but verification of this would require further investigation.

To show the functional dependence of $K^{*}$ on the other parameters, a series of plots of $K^{*}$ versus $K$ is given in figures la through $6 \mathrm{~b}$. The plots are arranged in pairs. Each pair contains plots having the same value of $K_{w}$. The first figure in the pair contains plots for different $D$ values running from $D=0.01$ to 0.07 , and the second contains these where $D=0.10$ to 1.00 . The six values of $K_{w}$ were $0.0,0.01,0.02,0.06,0.10$, and 0.20 . Values of $K^{*}$ corresponding to $K_{w}$ values lying between those given, can be obtained by a linear interpolation whose accuracy is better than $1 \%$. This probably exceeds the accuracy with which $K^{*}$ can be determined from the figures. The ranges covered by the parameters in these plots are the same as those given by (19) and should be sufficiently large to encompass the conditions encountered in the majority of low pressure, high flow velocity experiments.

\section{An Example Illustrating the Use of ROOT}

To demonstrate the use of ROOT, consider the following experiment. There is the reaction $A+B \rightarrow$ products, with a second order rate constant $k_{2}$ and with $[A]<<[B]$ so that a 
first order rate constant $k=k_{2}[B]$ can be defined. The reactants are contained in a carrier gas flowing through a 2 $\mathrm{cm} \mathrm{I.D.} \mathrm{tube} \mathrm{with} \mathrm{an} \mathrm{average} \mathrm{linear} \mathrm{velocity} \mathrm{of} 200 \mathrm{~cm} / \mathrm{s}$. The measurement of the concentration of $A$ is begun $5 \mathrm{~cm}$ downstream from its injection point and is found at $20 \mathrm{~cm}$ to have decayed exponentially to 0.1 of its value at $5 \mathrm{~cm}$. When species B is removed from the carrier, $A$ is observed at $20 \mathrm{~cm}$ to have decayed to 0.3 of its value at $5 \mathrm{~cm}$. Since $A$ decays in the absence of $B$, it is assumed to be undergoing a first order wall reaction. Thus, $k^{*}($ total $)=30.7 \mathrm{~s}^{-1}$, and $k^{*}($ wall $)=16.05 \mathrm{~s}^{-1}$. If the diffusion coefficient of $A$ in the carrier gas at the prevailing pressure is $20 \mathrm{~cm}^{2} \mathrm{~s}^{-1}$, then the values of the dimensionless parameters used by ROOT are, $K^{*}($ total $)=0.1535, K^{*}($ wall $)=0.0803$, and $D=$ 0.05 .

The first task is to use ROOT to determine $K_{w}$ from the value of $K^{*}$ observed in the absence of $B$. The following calling program will accomplish this.

PROGRAM CALL

DIMENSION B(30)

$\mathrm{ZS}=0.0803$

$\mathrm{Z}=0.0$

$\mathrm{D}=0.05$

$\mathrm{IOPT}=4$

CALL ROOT(ZS,Z,ZW,D,IOPT,F,B,NB,IFLAG)

PRINT ZW

END

In this case ROOT will return a value of 0.05 for $\mathrm{ZW}$ $\left(=K_{w}\right)$.

To determine $K$ from $K^{*}$ (total), use $K_{w}=0.05$, and use $1.2 K^{*}=0.1842$ as an approximate value of $K$. Then use ROOT with IOPT $=3$ to determine the correct value of $K$.
The following program accomplishes this.

PROGRAM CALL

DIMENSION $\mathrm{B}(30)$

$\mathrm{ZS}=0.1535$

$\mathrm{Z}=1.2 * \mathrm{ZS}$ (approximate value)

$\mathrm{ZW}=0.05$

$\mathrm{D}=0.05$

$\mathrm{IOPT}=3$

CALL ROOT(ZS,Z,ZW,D,IOPT,F,B,NB,IFLAG)

PRINT Z

END

For this example, ROOT gives 0.0842 for the accurate value of $K$.

From these results, the values, $k=16.84 \mathrm{~s}^{-1}$, and $k_{w}=$ $10.00 \mathrm{~cm} \mathrm{~s}^{-1}$ are obtained for the laboratory parameters.

It has been assumed in this experiment that the reactants are well mixed $5 \mathrm{~cm}$ downstream from the injection point of $A$. This is equivalent to assuming that only the leading term in (8) is being observed. ROOT can be used to determine the higher order decay parameters in (8). To do this for the above example, the values $K=0.0842, K_{w}=0.05$, and $D$ $=0.05$ are used with IOPT $=1$ to calculate $F$ as a function of $K^{*}$. From this the zero crossing points which correspond to higher roots of (13) can be located and used with IOPT = 2 to get accurate values. For the present example, the next decay parameter is $K_{2}^{*}=1.3144$, which yields $k_{2}^{*}=263$ $\mathrm{s}^{-1}$. Therefore, at $5 \mathrm{~cm}$ downstream from the mixing point, the second term in (8) would have decayed to 0.0014 of its initial value. This observation does not guarantee that only the leading term in (8) is being observed. If the coefficient $A_{2}$ were much larger than $A_{1}$ then the second term in (8)

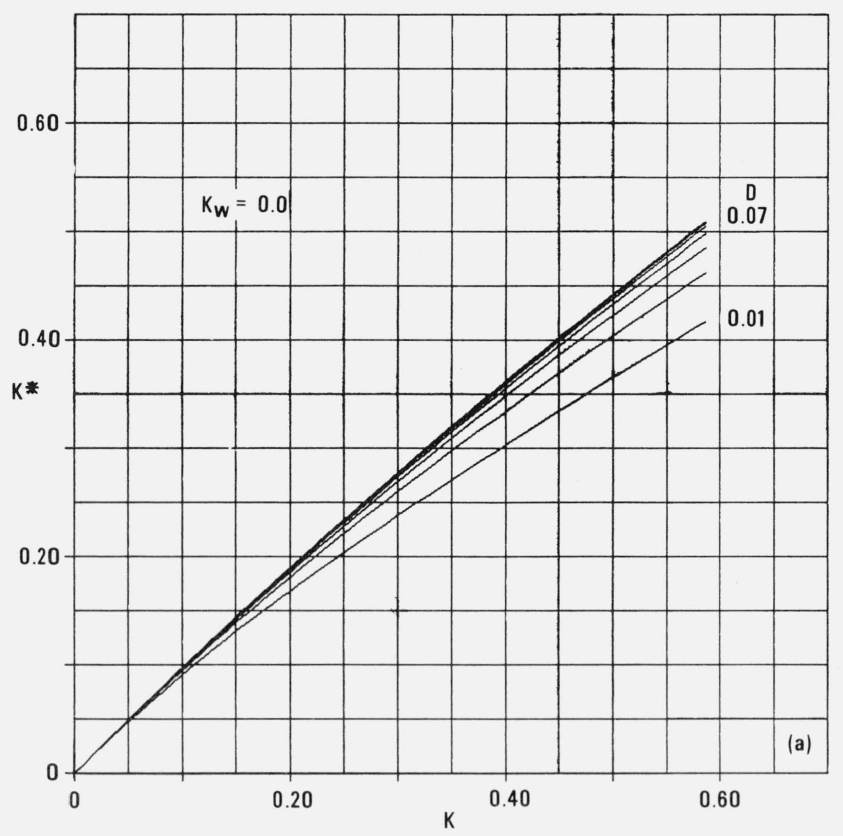

Figure la. Plots of $\mathrm{K}^{*}$ versus $\mathrm{K}$ for $\mathrm{D}=0.01,0.02,0.03,0.04,0.05$, $0.06,0.07$.

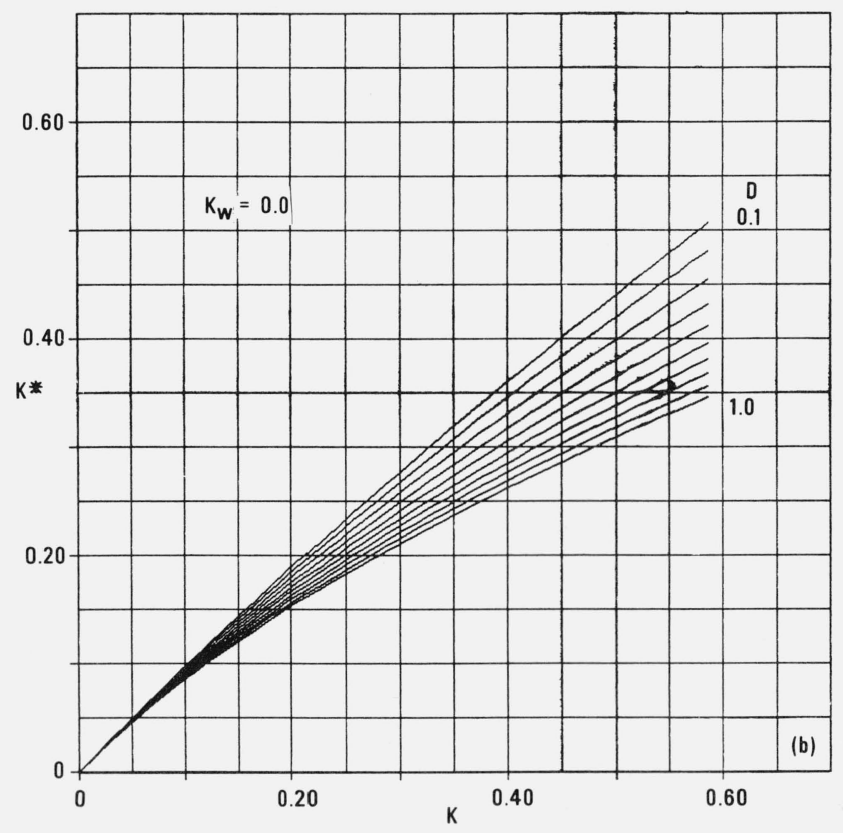

Figure 1b. Plots of $\mathrm{K}^{*}$ versus $\mathrm{K}$ for $\mathrm{D}=0.1,0.2,0.3,0.4,0.5,0.6$, $0.7,0.8,0.9,1.0$. $K_{w}=0.0$. 


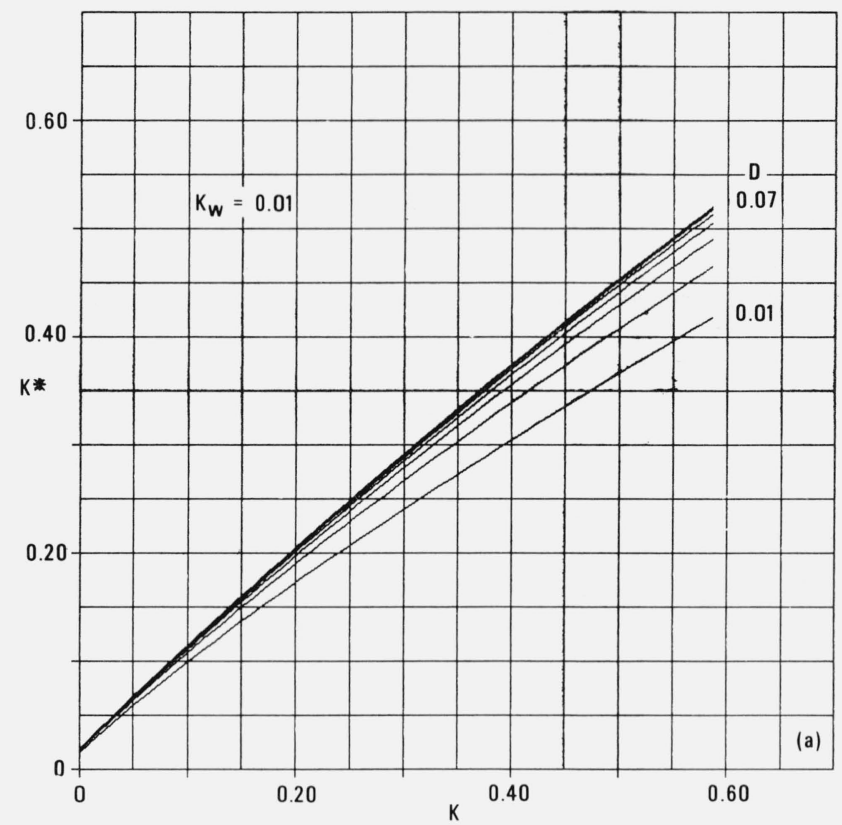

Figure 2a. Plots of $\mathrm{K}^{*}$ versus $\mathrm{K}$ for $\mathrm{D}=0.01,0.02,0.03,0.04,0.05$, $0.06,0.07$.
$K_{w}=0.01$.

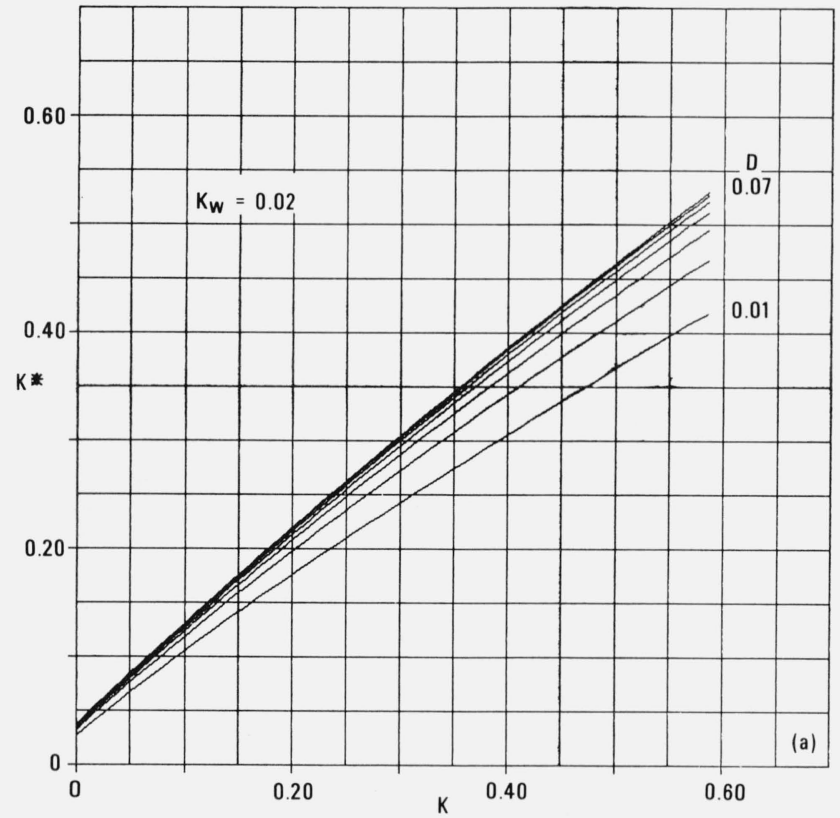

Figure 3a. Plots of $\mathrm{K}^{*}$ versus $\mathrm{K}$ for $\mathrm{D}=0.01,0.02,0.03,0.04,0.05$, $0.06,0.07$

$K_{w}=0.02$

could still be significant even though its exponential factor were small. As mentioned earlier, the coefficients $A_{i}$ could be determined from the radial distriubtion of $C$ at the mixing point. This distriubtion is, however, rarely known. Pirkle and Sigillito [7] have solved (4) for several cases in which the initial distribution of $C$ was uniform. In none of them did the values of $A_{i}, i \geqq 2$, exceed those of $A_{1}$. Unfortunately, it

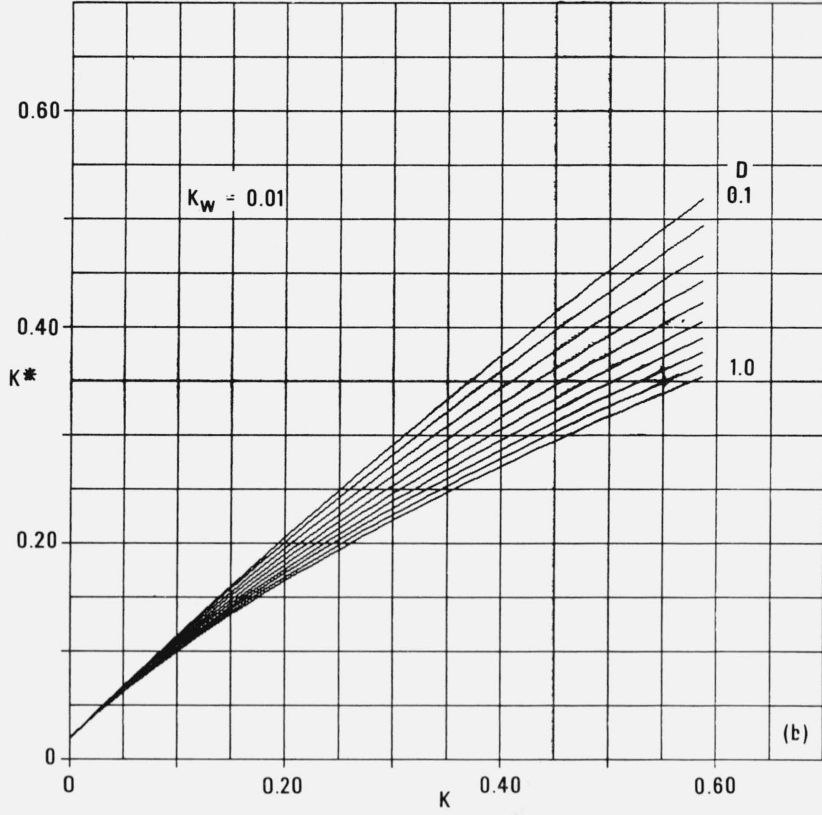

Figure 2b. Plots of $\mathrm{K}^{*}$ versus $\mathrm{K}$ for $\mathrm{D}=0.1,0.2,0.3,0.4,0.5,0.6$, $0.7,0.8,0.9,1.0$.

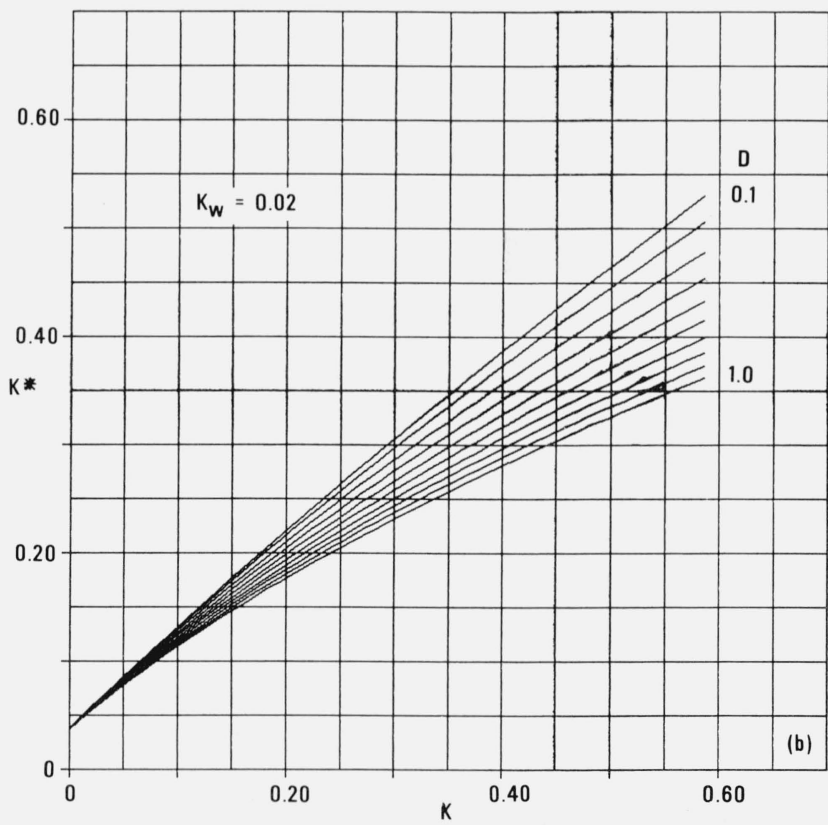

Figure $3 \mathrm{~b}$. Plots of $\mathrm{K}^{*}$ versus $\mathrm{K}$ for $\mathrm{D}=0.1,0.2,0.3,0.4,0.5,0.6$, $0.7,0.8,0.9,1.0$. $K_{w}=0.02$.

is difficult to say what the situation would be for other initial distributions. In practice, a logarithmic plot of the concentration versus distance is usually made and only the linear portion (within experimental error) is used to determine $k^{*}$. Such a procedure, coupled with an examination of the next higher decay parameters $k_{2}$ is most likely adequate to ensure observation of only the leading term in (8). 


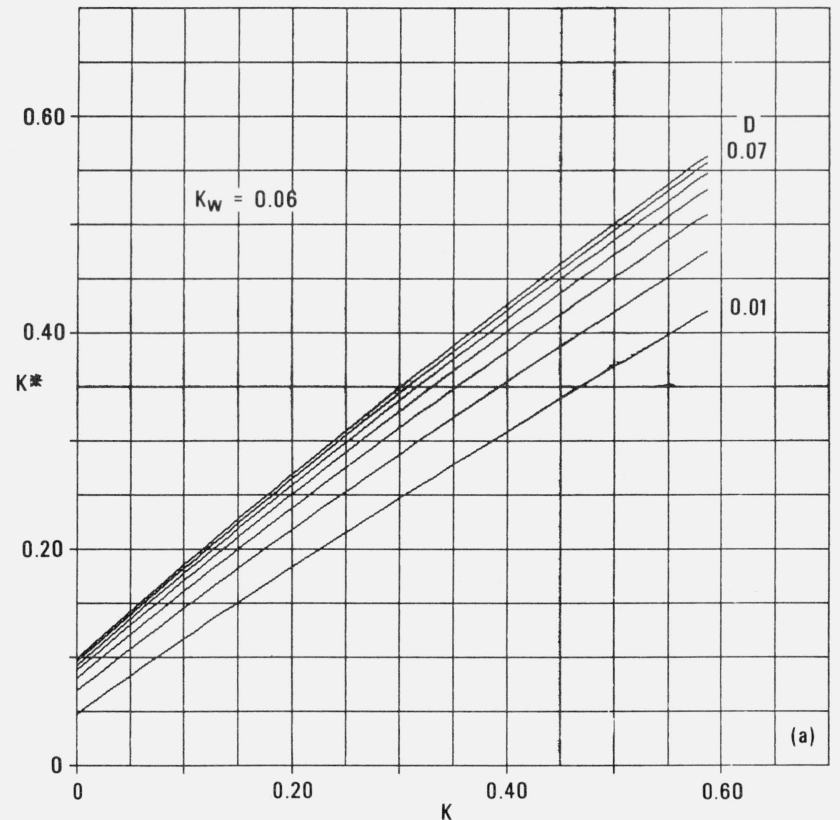

Figure 4a. Plots of $\mathrm{K}^{*}$ versus $\mathrm{K}$ for $\mathrm{D}=0.01,0.02,0.03,0.04,0.05$, $0.06,0.07$.

$K_{w}=0.06$

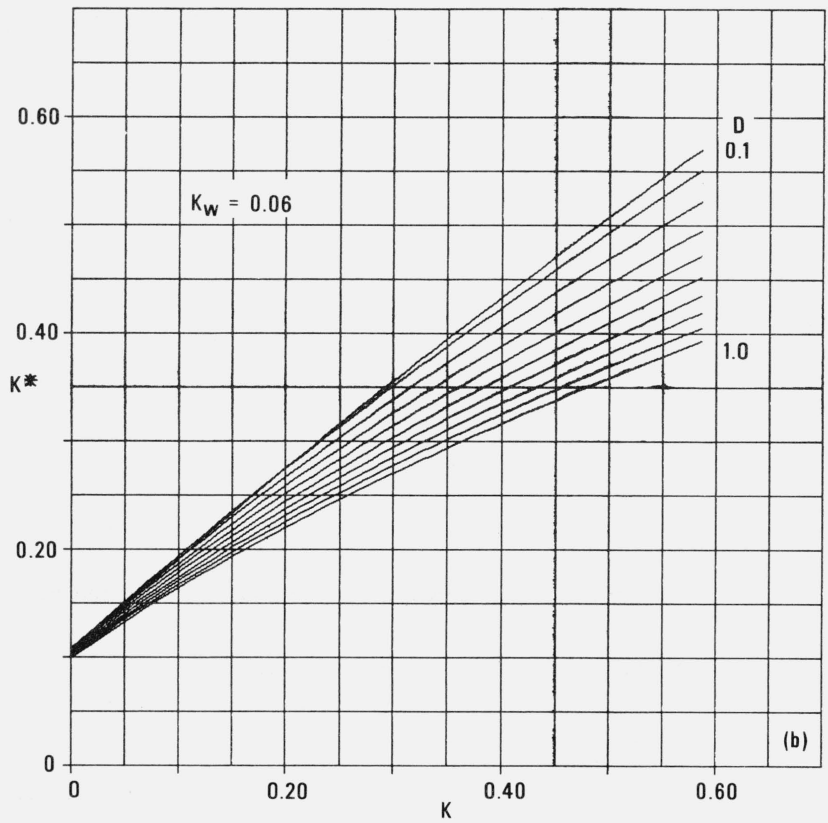

Figure 4b. Plots of $\mathrm{K}^{*}$ versus $\mathrm{K}$ for $\mathrm{D}=0.1,0.2,0.3,0.4,0.5,0.6$, $0.7,0.8,0.9,1.0$

$K_{w}=0.06$.

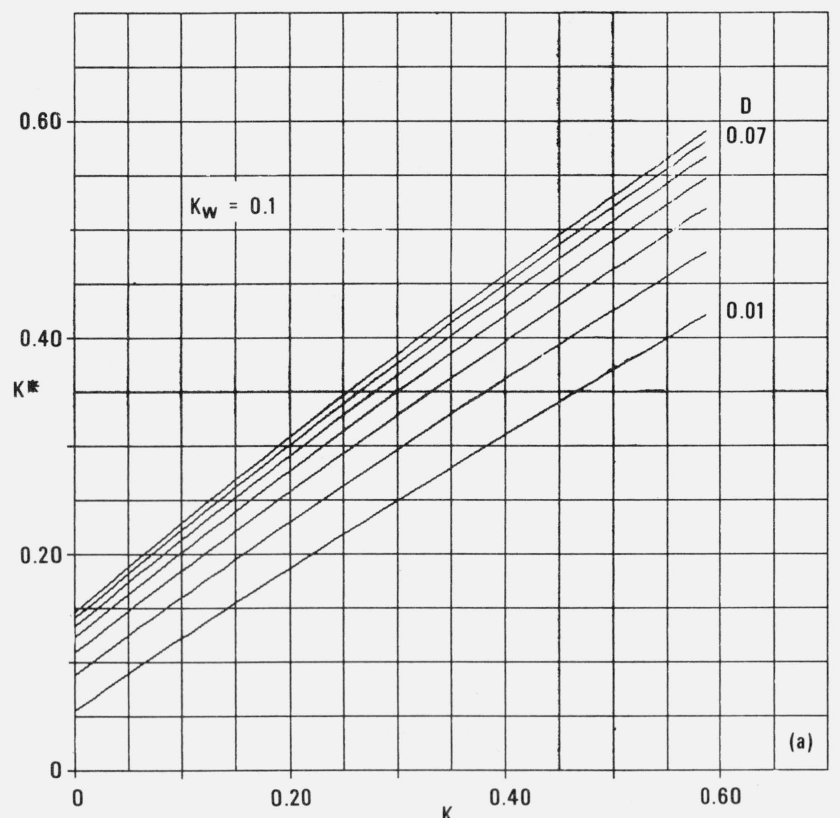

Figure 5a. Plots of $\mathrm{K}^{*}$ versus $\mathrm{K}$ for $\mathrm{D}=0.01,0.02,0.03,0.04,0.05$, $0.06,0.07$ $K_{w}=0.10$.

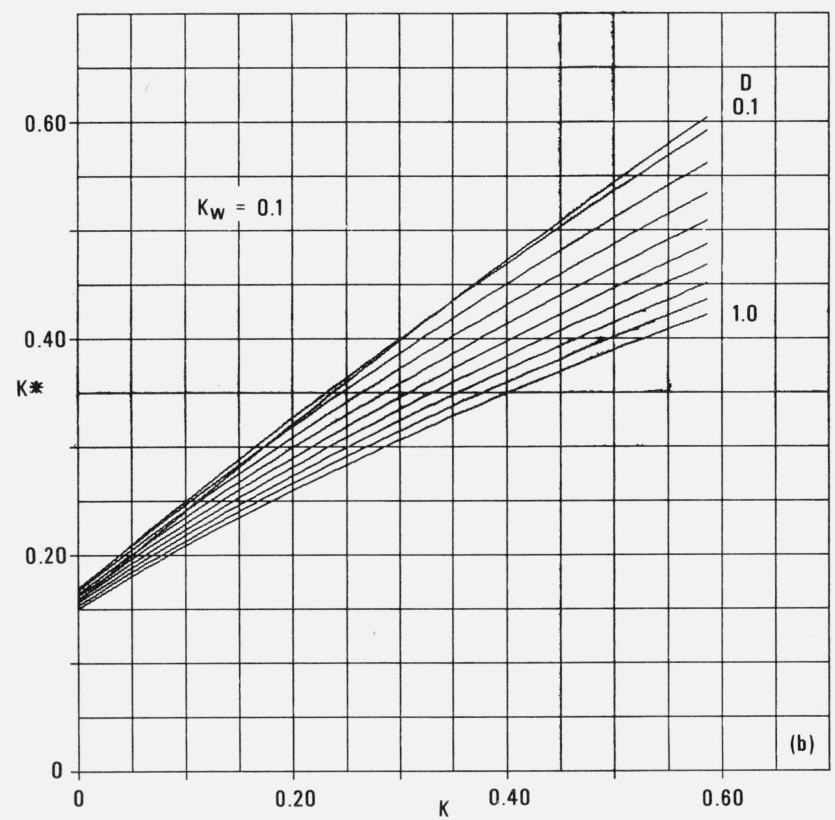

Figure 5b. Plots of $\mathrm{K}^{*}$ versus $\mathrm{K}$ for $\mathrm{D}=0.1,0.2,0.3,0.4,0.5,0.6$, $0.7,0.8,0.9,1.0$ $K_{w}=0.10$. 


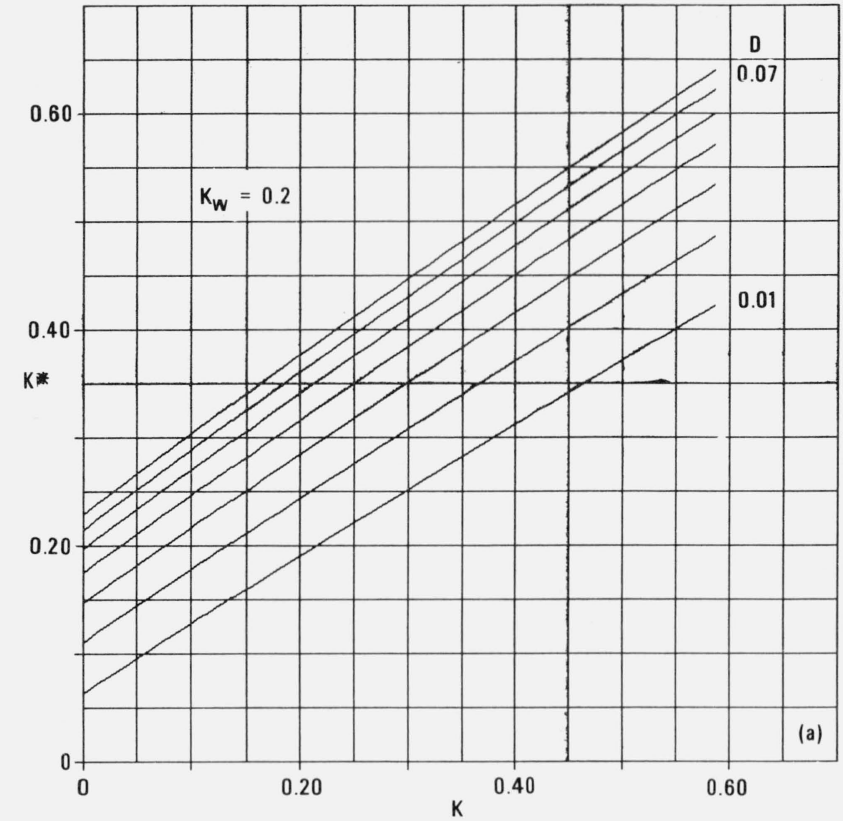

Figure 6a. Plots of $\mathrm{K}^{*}$ versus $\mathrm{K}$ for $\mathrm{D}=0.01,0.02,0.03,0.04,0.05$, $0.06,0.07$.

$K_{w}=0.20$

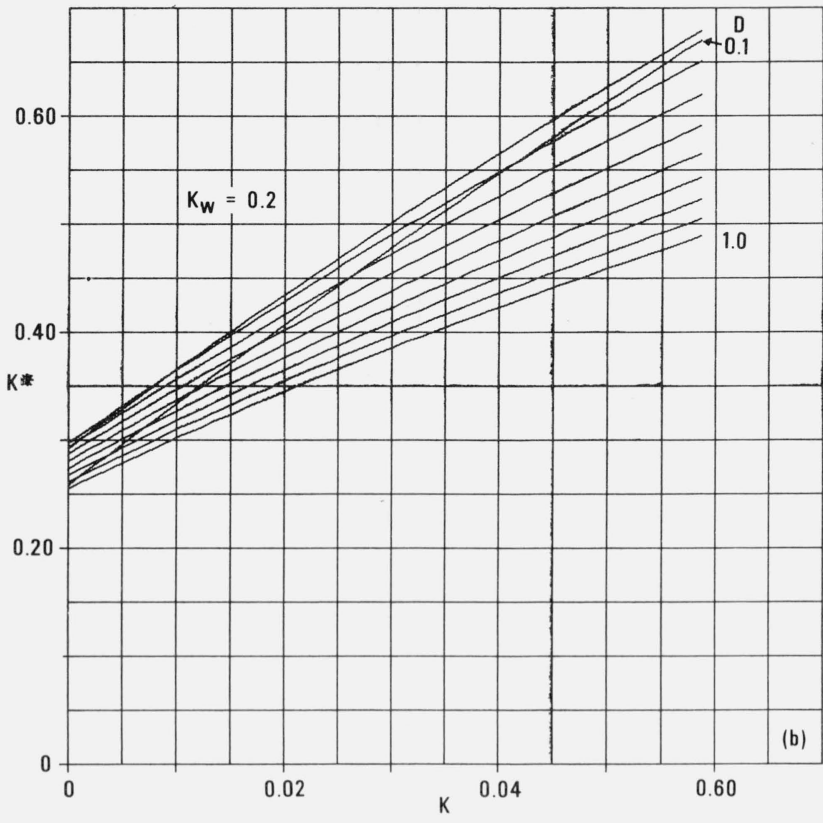

Figure 6b. Plots of $\mathrm{K}^{*}$ versus $\mathrm{K}$ for $\mathrm{D}=0.1,0.2,0.3,0.4,0.5,0.6$, $0.7,0.8,0.9,1.0$

$K_{w}=0.20$.

\section{Determination of $k$ in the Absence of $a$ Wall Reaction}

In the absence of any chemical reactions, Taylor [2, 3] and Aris [4] have shown that the coupling of axial and radial molecular diffusion with the parabolic velocity profile can be described by using the pseudo-diffusion coefficient $G$, given following (3), and considering only axial diffusion. The physical basis for the functional dependence of $G$ on the molecular diffusion coefficient $D_{c}$ can be seen by considering a species initially distributed in a radial plane moving with the average velocity of the carrier gas. As this distribution moves down the tube it will at first take the shape of a paraboloid. Molecules near the tube wall lag behind those at the center. However, molecular diffusion in the radial direction allows these molecules to move away from the wall into the faster flowing carrier. There will therefore be a net movement of the distribution down the tube. For small values of $D_{c}$, the distribution becomes dispersed about the radial plane just as though it were undergoing axial diffusion only, with a diffusion coefficient given by the second term in $G$. As $D_{c}$ becomes larger, the axial dispersion will decrease until a point is reached where true molecular axial diffusion surpasses the apparent axial diffusion resulting from the interaction of radial molecular diffusion with the velocity profile. Then the first term in $G$ becomes dominant. This is simply $D_{c}$, the molecular diffusion coefficient.

When the diffusing species is being destroyed by a first order gas phase reaction, it is a good approximation [8] to consider only axial diffusion and use $G$ for the diffusion coefficient. This yields the simple differential equation

$$
\langle u\rangle \frac{d C}{d z}=G \frac{d^{2} C}{d z^{2}}-k C .
$$

For the boundary conditions, $C(\mathrm{z}=0)=C_{0}$, and $C(z \rightarrow \infty)$ $\rightarrow 0$, the solution of this equation may be written as

$$
C=C_{0} e^{-k^{*} z /\langle u\rangle} .
$$

To establish the relationship between $k^{*}$ and $k$, evaluate the derivatives of $C$ from (2l) and substitute them along with (21) into (20). This yields the quadratic equation

$$
k^{*^{2}}+\left(\langle u\rangle^{2} / G\right) k^{*}-\left(\langle u\rangle^{2} / G\right) k=0 .
$$

Solving for $k^{*}$ gives

$$
k^{*}=1 / 2\left(-\langle u\rangle^{2} / G \pm \sqrt{ }\left\{\left(\langle u\rangle^{2} / G\right)^{2}+4\left(\langle u\rangle^{2} / G\right) k\right\}\right) .
$$

To get (23) into the form of (3), multiple it by $G /\langle u\rangle^{2}$. This yields

$$
G k^{*} /\langle u\rangle^{2}=1 / 2\left(-1 \pm \sqrt{ }\left\{1+4 G k /\langle u\rangle^{2}\right\}\right)=\lambda^{*} .
$$

Since $\lambda^{*}$ must be positive to satisfy the boundary condition $C(z \rightarrow \infty) \rightarrow 0$, the positive value of the square root must be used. The resulting expression is (3). Values of $k^{*}$ given by (3) were compared to the exact ones determined by Walker's method for the range of parameter values shown in figures la and $1 \mathrm{~b}$. The agreement averaged better than 0.2 percent over the whole range. 


\section{Appendix. Listing of Subroutine ROOT}

The program is shown here in single precision. To calculate decay parameters higher than $K_{3}^{*}$, rewrite it in double precision and increase the dimensions of the arrays $\mathrm{B}$ and $\mathrm{BN}$ to 40 .

SUBROUTINE ROOT $(Z S, Z, Z W, D, I O P T, F, B, N B, I F L A G)$

DIMENSION $\mathrm{B}(30), \mathrm{DB}(30)$

DFiTn PREC 1,PREC2, NMAX. IMAX/.0001,.0001,30,10/

IFLAG $=\varnothing$

$R 2 D=1 . /(2 . * D)$

$Z W D=Z ! W=R 2 D$

$\mathrm{RD}=1 . / \mathrm{D}$

ITAE $=0$

$702 S D=25 * R D$

$A=23 * 2 S+2 S D-2 * R 2 D$

IF(ILPT.EQ.2) DA=2.*ZS+RD

IFI IOPT, FO.3) $D A=-.5 * R D$

$B(1)=-.2 \Xi * \mathrm{~A}$

$D B(1)=-.25 * D A$

$B(2)=.9625)(2 S D-A * B(1))$

IF (IOPT.EQ.2) DB(2) $=.0525 *(R D-D A * E(1)-A * D E(1))$

IF (IOTT.EQ. 3) $D B(2)=.0625 *(-D A * B(1)-A * D B(1))$

$F=Z W D$

$\mathrm{DF}=, 0$

$\mathrm{GlY}=.0$

$\mathrm{CD}=1$.

JC $15 \mathrm{~N}=1.2$

Q $1=2$ WN

$Q 2=Q 1+Z W D$

IF (IOPT.EQ.4) GO TO 16

$\mathrm{F}=\mathrm{F}+\mathrm{B}(\mathrm{N}) * \mathrm{QQ2}$

IF ( (IOPT, EQ . 2) . OR ( IOPT $E Q, 3)) \quad D F=D F+D B(N) * Q 2$ GO TO 15

$16 G D=G D+E($ i $i)$

$G H=G(+B(N) * 01$

15 CONINUE

DO $18 \mathrm{~N}=3$. NMAX

$\mathrm{NE}=\mathrm{H}$

Q1 $=2 *+1$

$Q 2=01 * 21$

$\mathrm{RQ2}=1 . \mathrm{QQ2}$

$B(i)=R 02 *(2 S D * B(N-2)-A * B(N-1))$

G0 TO $(14,12,13,20)$, IOPT

$12 D S(H)=R Q 2 *(R D * B(N-2)+Z S D * D B(N-2)-D A * B(N-1)-A * D E(N-1))$ GO TC $1 \mathrm{i}$

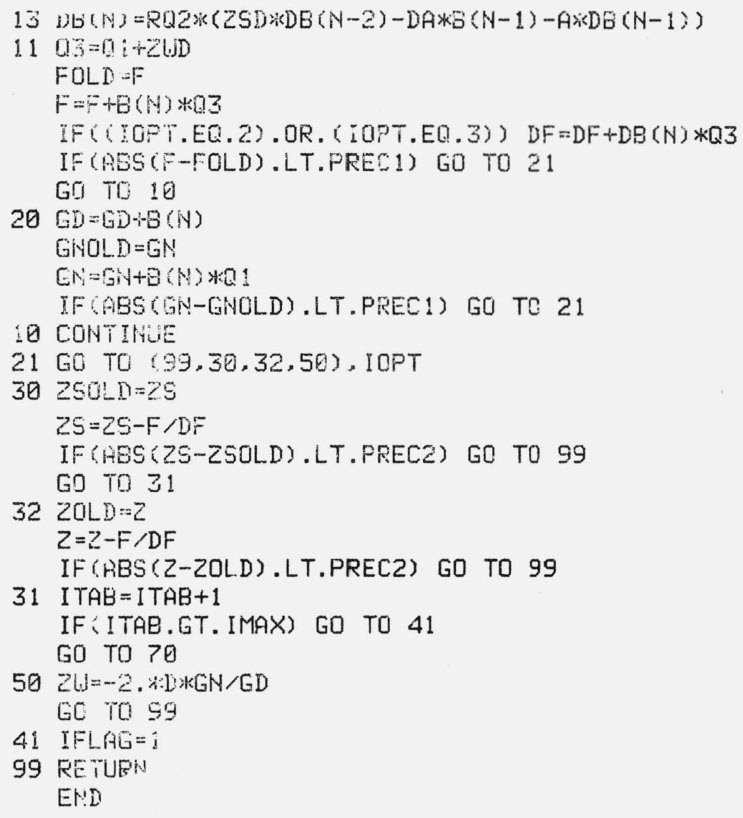

\section{References}

[1] Walker, R. E., Phys. Fluids 4, 1211 (1961).

[2] Taylor, G., Proc. Roy. Soc. A2 19, 186 (1953).

[3] Taylor, G., Proc. Roy. Soc. A225, 473 (1954).

[4] Aris, R., Proc. Roy. Soc. A235, 67 (1956).

[5] Dickens, P. G., Schofield, D., and Walsh, J., Trans. Faraday Soc. 56, $225(1960)$.

[6] Nielson, K. L., Methods in Numerical Analysis (MacMillan Co. New York, 1965).

[7] Pirkle, J. C., and Sigillito, V. C., Int. J. Engng Sci. 10, 553 (1972).

[8] Mulcahy, M. F. R., and Pethard, M. R., Aust. J. Chem. 16, 527 (1963). 